\title{
A SURFACE MICROMACHINED CAPACITIVE MICROPHONE FOR AEROACOUSTIC APPLICATIONS
}

\author{
D. T. Martin ${ }^{1}$, K. Kadirvel ${ }^{1}$, T. Nishida ${ }^{1}$, and M. Sheplak ${ }^{2}$ \\ ${ }^{1}$ Department of Electrical and Computer Engineering \\ ${ }^{2}$ Department of Mechanical and Aerospace Engineering \\ Interdisciplinary Microsystems Group, University of Florida, Gainesville, FL
}

\begin{abstract}
This paper presents improved results for a micromachined dual-backplate condenser microphone designed for aeroacoustic applications. Previous microphone characterization yielded an unacceptably high noise floor. This paper presents improvements to the noise floor through the use of a low-noise voltage amplifier and improved packaging. With bias voltages of $\pm 9.3 \mathrm{~V}$, an average sensitivity of $166 \mu \mathrm{V} / \mathrm{Pa}$ and a noise floor of $22.7 \mathrm{~dB} / \sqrt{ } \mathrm{Hz}$ for a 1 $\mathrm{Hz}$ bin centered at $1 \mathrm{kHz}$ are obtained. The noise floor is reduced to a level below that of other MEMS-based aeroacoustic microphones while maintaining sufficient bandwidth and maximum pressure.
\end{abstract}

\section{INTRODUCTION}

The goal of this research is to develop a microphone for aeroacoustic testing that equals the performance of conventional commercial measurement microphones. The applications for such a microphone include wind-tunnel testing using arrays consisting of a large number of microphones [1]. To be suitable for aeroacoustic measurements, a microphone must operate linearly up to a sound pressure level of $160 d B$ and have a bandwidth extending to $100 \mathrm{kHz}$. Furthermore, the noise floor should be as low as possible. The Brüel and Kjær $41381 / 8^{\text {th }}$ inch condenser microphone meets these requirements with a noise floor of 18 $\mathrm{dB} / \sqrt{\mathrm{Hz}}$ for a $1 \mathrm{~Hz}$ bin at $1 \mathrm{kHz}$ [2]. However, due to the high cost of these microphones, a low-cost MEMS microphone of adequate performance would enhance these measurements by allowing a greater number of microphones to be used.

There have been many MEMS microphones developed in the past; however, most of these have focused on audio applications [3]. While there has been limited development in the area of aeroacoustic microphones, their overall performance is far from that of the B\&K 4138 condenser microphone [4-7]. Several previous MEMS microphones have sufficient bandwidth and maximum pressure [4-6]; however, the noise floor of these devices is too high.

A dual-backplate capacitive microphone has been designed to meet the specifications for aeroacoustic measurements. Previously reported results for this microphone yielded an unacceptably high $41 \mathrm{~dB} / \sqrt{\mathrm{Hz}}$ noise floor [8]. This was obtained using a charge amplifier located far from the microphone, resulting in high parasitic capacitance. This paper presents results obtained with a low-noise voltage amplifier with packaging designed to reduce the parasitic capacitance.

\section{MICROPHONE STRUCTURE}

The dual-backplate capacitive microphone consists of three circular conducting plates. These plates are separated by two air gaps. Each layer is comprised of conductive doped-polysilicon, thus forming two capacitors. The outer plates are perforated with holes to allow the incident pressure to deflect the diaphragm. A photograph of the microphone is shown in Figure 1 and a schematic cross-section is shown in Figure 2.

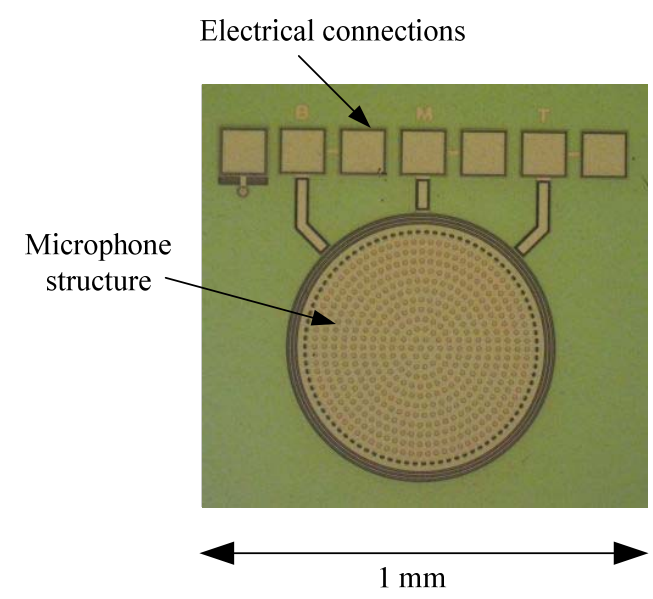

Figure 1: Photograph of the dual-backplate capacitive microphone.

The operation of this type of device is similar to that of the common single-backplate microphone. As the incident pressure impinges on the microphone, it deflects the diaphragm. This changes the values of the two capacitors causing one to increase while the other decreases. Bias voltages are applied to the two backplates, and the output is the voltage on the diaphragm.

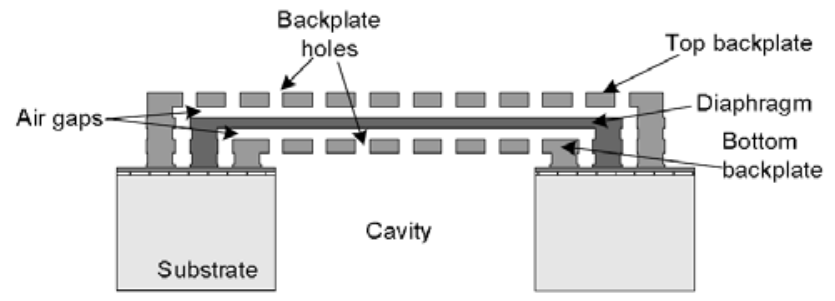

Figure 2: Cross-section of the dual-backplate capacitive microphone showing the key elements.

The microphone is fabricated using the SUMMiT V process at Sandia National Laboratories [9]. The 5-layer, planarized polysilicon process is well suited for this microphone. The conductive polysilicon is used to realize the three electrodes, and the use of chemical mechanical polishing (CMP) in the process results in uniform air gap spacing. This feature is also a major drawback of the process; the use of CMP results in high variability in the air gap thicknesses, which is manifested as a variability in device sensitivity [8]. A summary of the microphone physical specifications is given in Table 1.

\section{PACKAGING AND CIRCUITRY}

A schematic of the microphone and interface circuit is shown in Figure 3. The microphone, represented by $C_{1}$ and $C_{2}$, is biased with symmetric voltages $\pm V_{B}$, and the diaphragm electrode is connected to the voltage amplifier. Due to the small capacitance of this microphone, it is essential to minimize the capacitance load 
presented by the parasitic capacitance, $C_{p}$, and the amplifier input capacitance, $C_{i}$. As these capacitances increase, the sensitivity of the microphone is reduced [3].

Table 1: Microphone physical specifications.

\begin{tabular}{|l|c|}
\hline Property & Nominal value \\
\hline Diaphragm diameter & $460 \mu \mathrm{m}$ \\
\hline Diaphragm thickness & $2.25 \mu \mathrm{m}$ \\
\hline Air gap spacing & $2.0 \mu \mathrm{m}$ \\
\hline Top capacitance & $867 \mathrm{fF}$ \\
\hline Bottom capacitance & $632 \mathrm{fF}$ \\
\hline
\end{tabular}

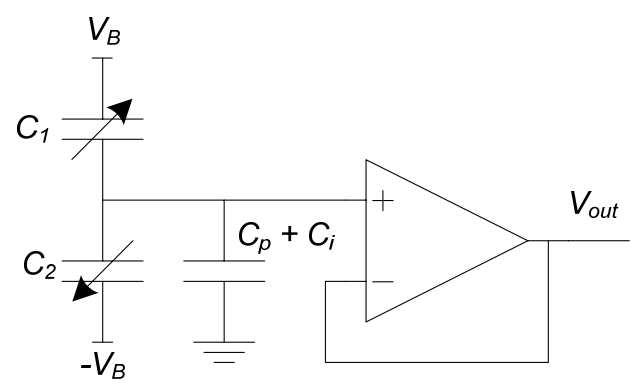

Figure 3: Circuit schematic showing the microphone and buffer amplifier.

To achieve minimal attenuation due to these capacitances, an amplifier with a very low input capacitance is used. The SiSonic Microphone Amplifier, courtesy of Knowles Electronics, is used for the interface circuitry. This amplifier has an input capacitance of approximately $0.3 p F[10]$.

The microphone package was designed to contain the amplifier and the MEMS die in close proximity to minimize parasitic capacitance introduced by cabling and electrical interconnect. This hybrid package was also designed to minimize interference with the acoustic field by mounting the microphone flush with the front surface of the package. A photograph of the assembled microphone package is shown in Figure 4. In sequence, the microphone is first mounted in a printed circuit board (PCB). The amplifiers are mounted on the reverse side of the circuit board. This preserves the flush surface, while minimizing the parasitic capacitance. This PCB is then secured in a Lucite block designed to be compatible with the acoustic test setup. Photographs of the front- and rear-views of the microphone package are shown in Figure 5. This shows the flush-mounted microphone die, which contains four microphones as well as several test structures. Gold wirebonds connect the MEMS die to the circuit board. These bond wires are covered in a protective epoxy.

The final package has a frontal surface area of $0.75 \mathrm{in} . \mathrm{x} 0.75$ in. This is considerably larger than an individual microphone. However, each die contains four microphones, thus the package contains four amplifiers. A smaller package footprint is possible for a re-designed die containing only one amplifier.

\section{EXPERIMENTAL RESULTS}

Previous results reported for the microphone employed a charge amplifier located off-package [8]. In this configuration, a sensitivity of $390 \mu \mathrm{V} / \mathrm{Pa}$ was achieved with a noise floor of 41 $d B / \sqrt{H z}$ at $1 \mathrm{kHz}$. The resonant frequency of the microphone was determined to be $178 \mathrm{kHz}$. The dominant source of noise for this initial characterization was due to the charge amplifier interface circuit. The results presented in this section show a significantly improved noise floor with a modest reduction in sensitivity.

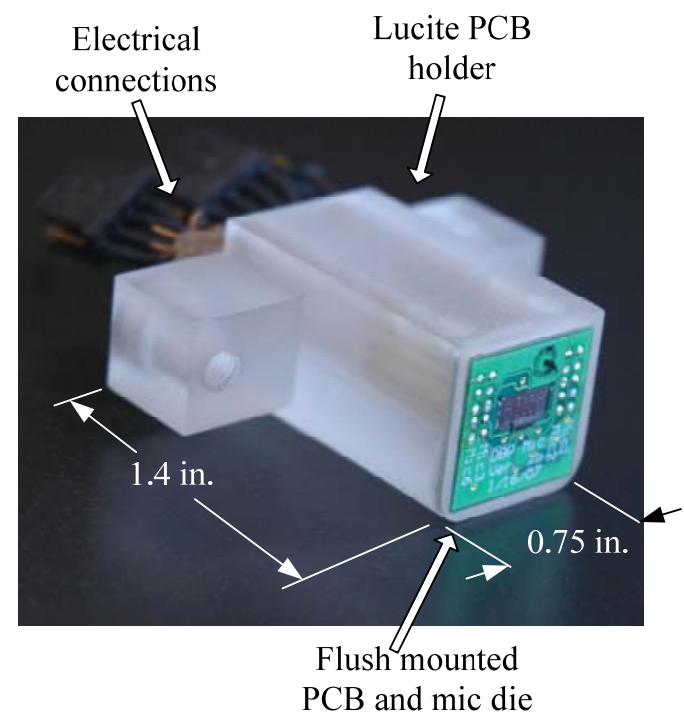

Figure 4: Photograph of the packaged microphone.
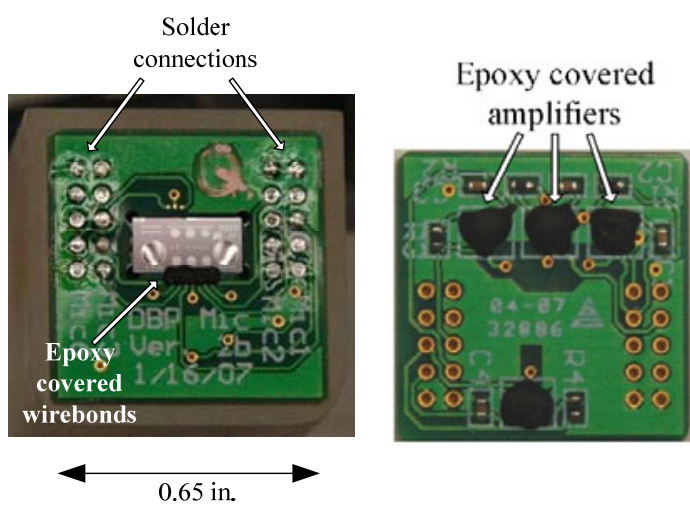

Figure 5: (Left) Front view of the microphone package showing the flush mounted MEMS die. (Right) Back view of the PCB showing four covered, mounted amplifier die.

A total of seven microphones are characterized with the improved circuitry. Experimentation is performed to determine the microphone linearity and frequency response. These acoustic tests are performed using a plane wave tube [11] with a maximum frequency of $20 \mathrm{kHz}$, as shown in Figure 6. The noise floor of the microphone is estimated by measuring the output of the microphone/amplifier system without an acoustic input. Bias voltages of $\pm 2 V$ are used unless otherwise specified. The experimental setups are described in detail by Martin et al. [8].

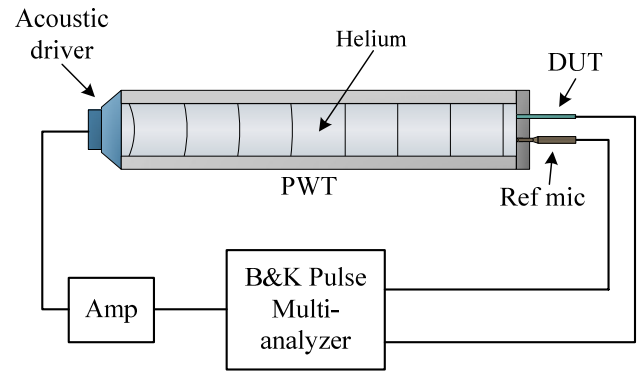

Figure 6: Experimental setup for acoustic experiments.

Figure 7 shows the output of the microphones for varying incident pressure. The amplitude of a $1 \mathrm{kHz}$ tone is varied from 
approximately $40 \mathrm{~dB}$ to $160 \mathrm{~dB}$ re. $20 \mu \mathrm{Pa}$. The output voltage of each microphone is plotted vs. the incident pressure. The dashed lines indicate the predicted output voltage. The average sensitivity is $166 \mu \mathrm{V} / \mathrm{Pa}$ with a variation of $\pm 20 \mu \mathrm{V} / \mathrm{Pa}$ between the seven microphones.

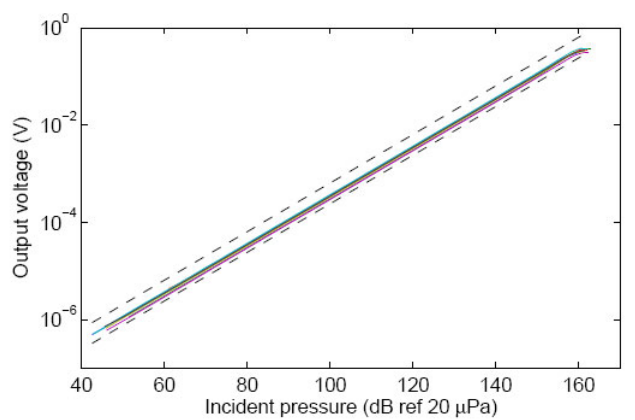

Figure 7: The output voltage of seven microphones vs. incident pressure at $1 \mathrm{kHz}$.

The microphone output saturates near $160 \mathrm{~dB}$; at this pressure, the microphone output voltage exceeds the input voltage of the amplifier. To quantify the distortion generated by the microphone, the bias voltage is reduced to $2 \mathrm{~V}$ to lower the sensitivity. This eliminates any distortion generated by the amplifier for the range of sound pressure levels tested. The total harmonic distortion of the seven microphones is given in Figure 8. The microphones show an average distortion of $4 \%$ at $164 d B$.

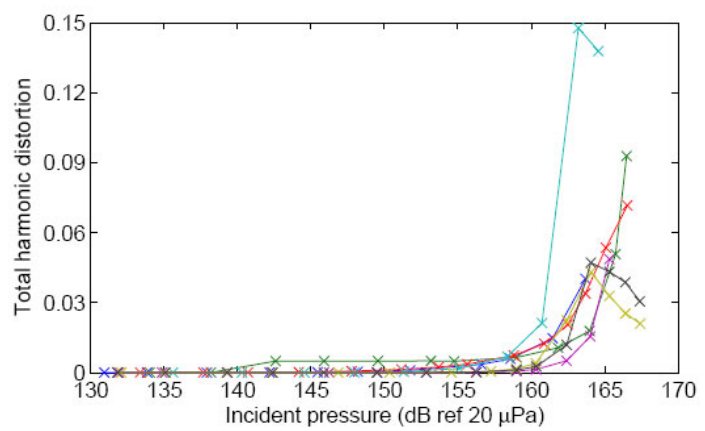

Figure 8: Total harmonic distortion of the seven microphones versus incident pressure at $1 \mathrm{kHz}$.

The frequency response of the seven microphones is measured from $300 \mathrm{~Hz}$ to $25 \mathrm{kHz}$. The magnitude response is plotted in Figure 9 and the phase response is plotted in Figure 10. The upper frequency for plane-waves in the acoustic waveguide is $20 \mathrm{kHz}$. Below this frequency, the device under test and a reference microphone are exposed to the same incident pressure. However, above this frequency, the two microphones are sensing a different pressure. Therefore, the magnitude response above 20 $\mathrm{kHz}$ is a qualitative measurement demonstrating microphone sensitivity up to this frequency. This measurement confirms the close sensitivity matching of the seven microphones.

The phase response shown in Figure 10 is plotted from 300 $\mathrm{Hz}$ to $20 \mathrm{kHz}$. For the majority of the frequency range, the phase is matched to within $\pm 1^{\circ}$. The dip near $10 \mathrm{kHz}$ corresponds to a reduced output of the acoustic driver. The cut-on of higher modes in the plane wave tube near $20 \mathrm{kHz}$ is evident in the phase response.

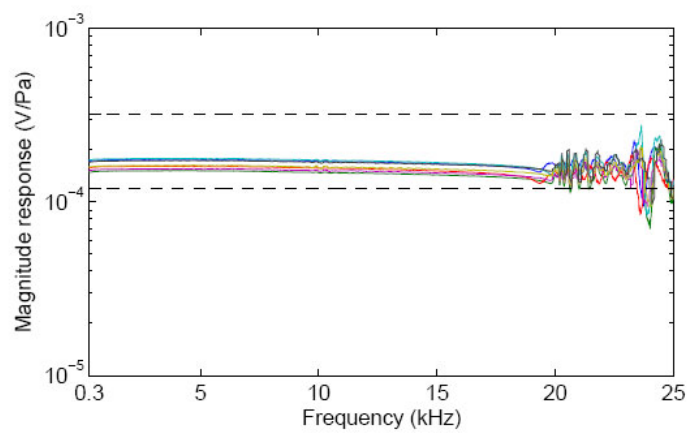

Figure 9: Magnitude response plotted over the range of $300 \mathrm{~Hz}$ to $25 \mathrm{kHz}$.

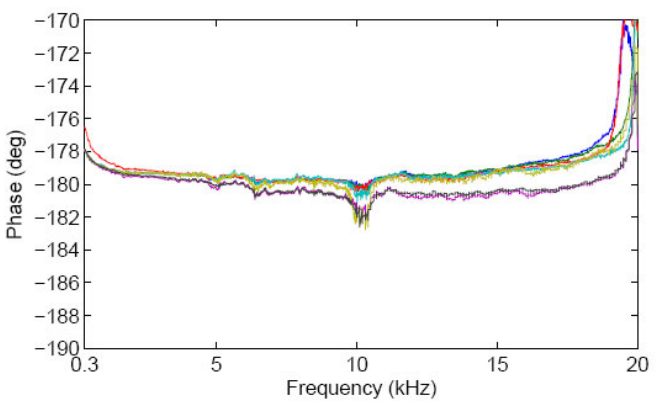

Figure 10: Phase response of the seven microphones plotted over the range of $300 \mathrm{~Hz}$ to $20 \mathrm{kHz}$.

The final measurements performed on the microphones with the improved circuitry determined the noise. The packaged microphones were placed in a Faraday cage to reduce the effects of electromagnetic interference [12]. With no acoustic input to the microphone, the output noise voltage power spectral density (PSD) of the amplifier was measured with a spectrum analyzer over the range of $10 \mathrm{~Hz}$ to $100 \mathrm{kHz}$. Dividing the square root of the PSD by the microphone sensitivity yields the input referred noise, plotted in Figure 11. For a $1 \mathrm{~Hz}$ bin centered at $1 \mathrm{kHz}$, the average noise level of the seven microphones is $22.7 \mathrm{~dB} / \sqrt{ } \mathrm{Hz}$. The input referred noise for the seven microphones falls within $\pm 1 d B$. Table 2 shows the average noise floor in several equivalent units including a minimum detectable force and capacitance change. The average A-weighted noise figure for the seven microphones is $60.4 \mathrm{dBA}$.

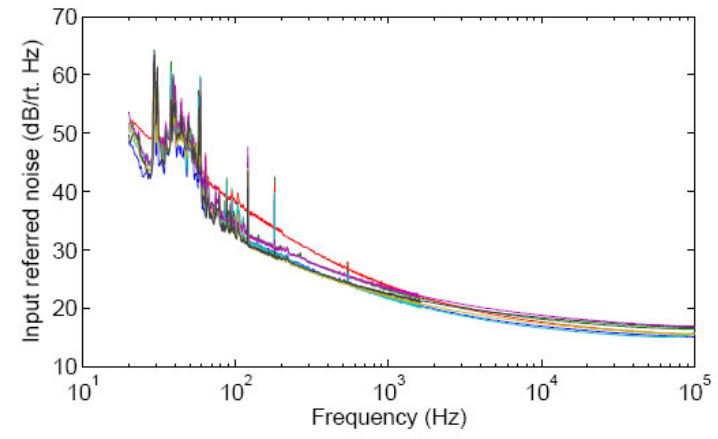

Figure 11: Input referred noise spectrum of the seven microphones.

These results demonstrate the importance of the interface circuitry on the performance of the capacitive MEMS microphone. The lower noise voltage amplifier and hybrid package reduced the noise floor by $18 d B$. This improvement was due to a large 
reduction in the amplifier noise. The acoustic noise generated by the microphone structure was less significant than the electronic noise [8].

Table 2: The average minimum detectable signal of the seven microphones expressed in several equivalent units at $1 \mathrm{kHz}$ in a 1 $\mathrm{Hz}$ bin.

\begin{tabular}{|l|c|}
\hline $\begin{array}{l}\text { Average Minimum } \\
\text { detectable Signal }\end{array}$ & Value \\
\hline Pressure & $22.7 \mathrm{~dB}$ \\
\hline Pressure & $273 \mu \mathrm{Pa}$ \\
\hline Force & $15.1 \mathrm{pN}$ \\
\hline Displacement & $75.4 \mathrm{fm}$ \\
\hline Capacitance & $17.9 \mathrm{zF}$ \\
\hline
\end{tabular}

The performance of the dual-backplate capacitive microphone is compared to other MEMS microphones, as well as the Brüel and Kjær 4138 condenser microphone in Table 3. The other microphones are sufficient in two specifications listed; however, all fall short of the B\&K 4138 in at least one. The present work exceeds the performance of the other MEMS microphones and compares most favorably to the commercial microphone. It is within $4 d B$ of the 4138's maximum pressure and $3 d B$ of the noise floor. However, the diameter of the dual-backplate microphone is significantly smaller. With the appropriate packaging, this device has the potential to enable microphone placement in locations prohibited by the size of the B\&K 4138 microphone.

Table 3: Comparison of the present work to other aeroacoustic microphones.

\begin{tabular}{|l|c|c|c|c|}
\hline Microphone & Diameter & $\begin{array}{c}\text { Max } \\
\text { Pressure }\end{array}$ & $\boldsymbol{f}_{\max }$ & $\begin{array}{c}\text { Noise } \\
\text { Floor }\end{array}$ \\
\hline Present Work & $460 \mu \mathrm{m}$ & $164 \mathrm{~dB}$ & $178 \mathrm{kHz}$ & $22.7 \mathrm{~dB}^{1}$ \\
\hline B\&K 4138. ${ }^{[2]}$ & $3.2 \mathrm{~mm}$ & $168 \mathrm{~dB}$ & $140 \mathrm{kHz}$ & $18 \mathrm{~dB}^{1}$ \\
\hline Arnold et al. $^{[4]}$ & $1.0 \mathrm{~mm}$ & $160 \mathrm{~dB}$ & $100 \mathrm{kHz}$ & $52 \mathrm{~dB}^{1}$ \\
\hline Scheeper et al. $^{[5]}$ & $3.9 \mathrm{~mm}$ & $141 \mathrm{~dB}$ & $20 \mathrm{kHz}$ & $23 \mathrm{dBA}$ \\
\hline Horowitz et al. $^{[6]}$ & $1.8 \mathrm{~mm}$ & $169 \mathrm{~dB}$ & $59 \mathrm{kHz}$ & $35.7 \mathrm{~dB}^{1}$ \\
\hline Pedersen $^{[7]}$ & $360 \mu \mathrm{m}$ & $140 \mathrm{~dB}$ & $75 \mathrm{kHz}$ & $22 \mathrm{~dB}^{1}$ \\
\hline
\end{tabular}

${ }^{1}$ Noise figure at $1 \mathrm{kHz}$ in a $1 \mathrm{~Hz}$ bin

\section{CONCLUSIONS}

An instrumentation-grade dual-backplate capacitive microphone has been designed for aeroacoustic measurements. Improvements in the packaging and the use of a low-noise voltage amplifier significantly improved the device performance over the initial characterization results. The noise floor has been reduced while maintaining adequate bandwidth and maximum pressure. The device characterization demonstrates performance that exceeds that of existing MEMS aeroacoustic microphones. In addition, the dual-backplate capacitive microphone compares favorably to the Brüel and Kjær 4138 condenser. For applications not requiring measurements at the extreme ends of the 4138's dynamic range, the designed MEMS microphone is suitable.

\section{ACKNOWLEDGMENTS}

Financial support for this project was provided by the National Science Foundation grant \#ECS-0097636 and Sandia National Laboratories. The authors gratefully acknowledge Dr. Pete Loeppert, from Knowles Electronics, for providing the SiSonic microphone amplifier used as part of this work.

\section{REFERENCES}

[1] T. J. Mueller, Aeroacoustic Measurements. Berlin, Germany: Springer-Verlag, 2002, pp. 158-179.

[2] Brüel and Kjær, Product Data, Condenser Microphone Cartridges-Types 4133 to 4181, p. 6. [Online]. Available: http://www.bksv.com/pdf/Bp0100.pdf

[3] P. R. Scheeper, A. G. H. van der Donk, W. Olthuis, and P. Bergveld, “A review of silicon microphones," Sens. Actuators A, Phys., vol. 44, no. 1, pp. 1-11, Jul. 1994.

[4] D. P. Arnold, S. Gururaj, S. Bhardwaj, T. Nishida, and M. Sheplak, "A piezoresistive microphone for aeroacoustic measurements,” in Proc. ASME IMECE, 2001, pp. 281-288.

[5] P. R. Scheeper, B. Nordstrand, B. L. J. O. Gullov, T. Clausen, L. Midjord, and T. Storgaard-Larsen, "A new measurement microphone based on MEMS technology," J. Microelectromech. Syst., vol. 12, no. 6, pp. 880-891, Dec. 2003.

[6] S. Horowitz, T. Nishida, L. Cattafesta, and M. Sheplak, "Development of a micromachined piezoelectric microphone for aeroacoustic applications J. Acoust. Soc. Amer, Vol. 122, No. 6, 2007, pp. 3428-3436.

[7] M. Pedersen, "Development of microelectromechanical systems capacitive microphone for high-frequency applications," in Proc. of 151st Meeting of the Acoust. Soc. Amer. Providence, RI, 2006.

[8] D. T. Martin, J. Liu, K. Kadirvel, R. M. Fox, M. Sheplak, and T. Nishida, "A Micromachined Dual-Backplate Capacitive Microphone for Aeroacoustic Measurements," Journal of Microelectromechanical Systems, Vol. 16, No. 6, 2007, pp. 1289-1302

[9] J. J. Sniegowski and M. S. Rodgers, "Multi-layer enhancement to polysilicon surface-micromachining technology,” in Proc. IEDM Tech. Dig., 1997, pp. 903-906.

[10] P. V. Loeppert and S. B. Lee, "SiSonic-The first commercialized MEMS microphone," in Proc. Solid-State Sensor Actuator Workshop, Hilton Head Island, SC, 2006, pp. 7-30.

[11] T. Schultz, L. Cattafesta, and M. Sheplak, "Modal decomposition method for acoustic impedance testing square ducts,” J. Acoust. Soc. Amer., vol. 120, no. 6, pp. 3750-3758, Dec. 2006.

[12] R. Dieme, G. Bosman, M. Sheplak, and T. Nishida, "Source of excess noise in silicon piezoresistive microphones," J. Acoust. Soc. Amer., vol. 119, no. 5, pp. 2710-2720, 2006. 